\title{
Genome-Wide DNA Methylation Profiles of Phlegm-Dampness Constitution
}

\author{
Haiqiang Yao Shanlan Mob,c Ji Wanga Yingshuai Lia Chong-Zhi Wang ${ }^{\mathrm{a}}$ \\ Jin-Yi Wan ${ }^{\mathrm{a}, \mathrm{d}}$ Zengliang Zhang ${ }^{\mathrm{a}}$ Yu Chen ${ }^{\mathrm{a}}$ Ranran Sun ${ }^{\mathrm{a}}$ Chun-Su Yuan ${ }^{\mathrm{d}}$ \\ Xin Liub,c Lingru Lia ${ }^{\mathrm{a}} \quad$ Qi Wang ${ }^{\mathrm{a}}$
}

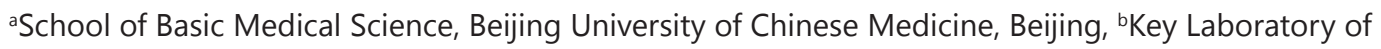
Genomic and Precision Medicine, Beijing Institute of Genomics, Chinese Academy of Sciences, Beijing, 'University of Chinese Academy of Sciences, Beijing, China; ${ }^{d}$ Tang Center for Herbal Medicine Research and Department of Anesthesia \& Critical Care, Pritzker School of Medicine, University of Chicago, Chicago, USA

\section{Key Words}

Dna methylation - Peripheral blood mononuclear cells • Bioinformatics $\cdot$ Chinese medicine $•$ Phlegm-dampness constitution

\begin{abstract}
Background/Aims: Metabolic diseases are leading health concerns in today's global society. In traditional Chinese medicine (TCM), one body type studied is the phlegm-dampness constitution (PC), which predisposes individuals to complex metabolic disorders. Genomic studies have revealed the potential metabolic disorders and the molecular features of PC. The role of epigenetics in the regulation of PC, however, is unknown. Methods: We analyzed a genome-wide DNA methylation in 12 volunteers using Illumina Infinium Human Methylation450 BeadChip on peripheral blood mononuclear cells (PBMCs). Eight volunteers had PC and 4 had balanced constitutions. Results: Methylation data indicated a genome-scale hyper-methylation pattern in PC. We located 288 differentially methylated probes (DMPs). A total of 256 genes were mapped, and some of these were metabolic-related. SQSTM1, DLGAP2 and DAB1 indicated diabetes mellitus; HOXC4 and SMPD3, obesity; and GRWD1 and ATP10A, insulin resistance. According to Ingenuity Pathway Analysis (IPA), differentially methylated genes were abundant in multiple metabolic pathways. Conclusion: Our results suggest the potential risk for metabolic disorders in individuals with PC. We also explain the clinical characteristics of PC with DNA methylation features.
\end{abstract}

\section{Introduction}

Due to widespread over-nutrition and sedentary lifestyles, metabolic diseases and the subsequent morbidity and mortality are major public health issues in our current H. Yao and S. Mo contributed equally to this work.

Lingru Li

and Qi Wang
School of Basic Medical Science, Beijing University of Chinese Medicine, Beijing (China) Tel. +86 10 64286766, E-Mail lilingru912@163.com, wangqi710@126.com 
society [1]. The steadily increasing prevalence of metabolic disease may be associated with cardiovascular disease and diabetes [2]. It has been estimated that $25 \%$ of the world's adult population is suffering from these diseases, and these have notoriously emerged as being the world's major medical and financial burdens [3]. In addition to conventional therapy, early prevention and prediction are beneficial in the promotion of health optimization according to "P4 medicine" [4].

Increasing evidence has revealed that genetic, environmental and dietary factors contribute to the development of metabolic diseases. Epigenetics is thought to be a potential link between postnatal environmental factors and diseases, which refers to a reversible and heritable change that regulates gene expression without a change in the DNA sequence. DNA methylation, as a major epigenetic mechanism that typically occurs at CpG sites in adult cells, is frequently studied [5-9]. Due to its stability in blood samples and transmissibility during cell division, DNA methylation can be used to characterize early disease progression, and it provides an efficient way to prevent and predict diseases in clinical practice. To date, changes in DNA methylation have been widely reported to be involved in the onset of a variety of metabolic diseases, including diabetes mellitus, fatty liver, and metabolic syndrome [10-15].

Phlegm-dampness constitution (PC), one of the nine different body constitution types in Traditional Chinese medicine (TCM), is thought to be the preclinical stage of multiple metabolic disorders $[16,17]$. Our previous studies have mainly focused on genomics and single nucleotide polymorphisms (SNPs) of PC, and we discovered correlations between the PC and multiple metabolic disorders $[16,18]$. However, there have been no epigenetic studies on PC formation, particularly the effects of DNA methylation. As an ongoing study, we aim to investigate the DNA methylation profile of PC on a genome-wide level using the balanced constitution (BC) as a control group. We also hope to evaluate the difference between the two constitution types (BC and PC) to discover the potential molecular biomarkers of PC. This study could reveal the molecular mechanism behind the preclinical stage of metabolic diseases and shed new light on early prevention and prediction.

\section{Materials and Methods}

\section{Study subjects}

Eight PC volunteers and four BC volunteers were included according to the standard of Classification and diagnosis of TCM Constitution [19]. The participants ranged in age from 30 to 60 years old. They had no diagnosed diseases, and all of them provided written informed consent. This study was approved by the Beijing University of Chinese Medicine ethics committee and performed in accordance with the Declaration of Helsinki.

\section{PBMC isolation}

Venous blood samples were extracted with an EDTA anticoagulant tube. Peripheral blood mononuclear cells (PBMCs) were isolated using Ficoll-Hypaque (Histopaque-1077, Sigma-Aldrich, St. Louis, USA) density gradient centrifugation.

\section{DNA extraction}

Genomic DNA was extracted from the isolated cell pellets using DNeasy Blood and Tissue Kit (Qiagen, Hilden, Germany) according to the manufacturer's instructions. DNA purity and quantity was assessed using a NanoDrop Spectrophotometer (Thermo Scientific, DE, USA), and the samples were diluted to standard concentrations of $50 \mathrm{ng} / \mu \mathrm{L}$.

\section{Infinium 450K BeadChip analysis}

Bisulfite conversion was conducted on $1 \mu \mathrm{g}$ of DNA sample from each participant using the EZ DNA Methylation kit (Zymo Research Corp, Orange, CA, USA). A genome-wide DNA methylation examination was performed using the Infinium Human Methylation450 BeadChip (Illumina, San Diego, CA, USA), which interrogates 485, 512 CpG sites [20, 21]. BeadChips were scanned with an Illumina iScan; the scanned data and image output files were managed with Genomestudio software (version 1.9.0; Illumina). 


\section{Bioinformatics and Statistical Analysis}

Illumina $450 \mathrm{~K}$ probe preprocessing. No outlier samples were identified after comparing the methylation beta value box plot and density plot of 12 subjects. All samples were included in the subsequent analysis. At first, we excluded 144, 854 CpG probes that ambiguously mapped to the human genome (hg19), and a total of 340, 658 probes passed the analysis using Bowtie [22]. We also filtered 12, $871 \mathrm{CpG}$ probes that contained common single-nucleotide polymorphisms (SNPs) so that the relative methylation level in each sample was not affected by potential genetic bias. Next, we removed 3965 probes that had a bead count $<3$ in $5 \%$ of samples or $>1 \%$ of samples with a detection $p$-value of $>0.01$. The residual signal intensity values were normalized using the subset-quantile within an arrays normalization (SWAN) method for reducing technical variation, as implemented in the minfi R-package [23]. Although one chip is not irrespective of the batch effect in our data, we excluded $7466 \mathrm{CpG}$ sites on the X-and Y-chromosomes to reduce the effects of differential methylation between males and females on the sex chromosomes. WBC proportion estimation for each sample was necessary for controlling for the effects of the relative proportions of different WBC types on methylation level. In the end, a total of 316, 356 autosomal CpG sites were tested in all samples (Fig. 1A).

To identify differentially methylated CpG sites. The beta value indicated the DNA methylation level of each CpG probes, and beta was defined as: Beta=Meth/ (Meth+Unmeth+100). However, since the beta value was in a proportion range from 0 to 1 , we transformed it to $M$ value, which was defined as: $M=\log$ (beta/ (1-beta)) [24]. Differentially methylated probes (DMPs) were detected by comparing the M values between the two groups. Statistical significance was tested with an unpaired Student's t-test and Wilcoxon signed rank test. Due to the small sample size of this study, we analyzed the data using the two statistical methods separately and identified the probes that overlapped as the DMPs. Finally, the analytic approach we adopted to rank DMPs was to take both statistical significance ( $p$-value) and the magnitude of absolute mean beta value difference (i.e., absolute $\Delta \beta$ ).

IPA analysis. The Ingenuity Pathway Analysis (IPA) system was used to analyze potentially altered network relationships, candidate biomarkers, and associated diseases from the list of differentially methylated genes between PC and BC individuals. Using a nonparametric test, IPA measured the likelihood due to chance that genes from the list participated in each network and category of Molecular Function and diseases and calculated the corresponding p-value.

\section{Results}

\section{Subjects' characteristics}

The characteristics of the study subjects in the PC and BC group are shown in Table 1. No significant differences were noted between these two groups in sex, age, body mass index (BMI), waist circumference, fasting blood glucose, fasting insulin, blood pressure, total cholesterol, triglyceride, low-density lipoprotein, or high-density lipoprotein. All subjects from both groups were healthy individuals with no diagnosable diseases.

\section{Identification of global methylation patterns in $P C$ and $B C$ subjects}

A total of 316, 356 sites on the beadchip were included after probe filtering and normalization (Fig. 1A). A genome-wide DNA methylation analysis was performed to explore the global methylation pattern of PC and $\mathrm{BC}$ subjects. Methylation levels of CpG sites in the two groups were strongly correlated as shown in Fig. 1B (Pearson's $\mathrm{R}^{2}=0.9978715$, $\mathrm{p}$-value $<2.2 \mathrm{e}-$ Table 1. Characteristics of the study subjects. ${ }^{*}$ P-values were calculated respectively using Student's t-test and Fisher's exact test for continuous and categorical variables

\begin{tabular}{lccc}
\hline Variables & Phlegm-dampness Constitution & $\begin{array}{c}\text { Balanced Constitution } \\
(\mathrm{N}=4)\end{array}$ & P-Value* \\
\hline Male, $\mathrm{n}(\%)$ & $2(25)$ & $1(25)$ & 1 \\
Age (years),Mean (SD) & $44.63 \pm 3.640$ & $35.25 \pm 2.658$ & 0.1231 \\
Body mass index (kg/m2),mean(SD) & $23.44 \pm 0.6068$ & $23.55 \pm 0.5008$ & 0.9072 \\
Waist circumference (cm),mean(SD) & $80.50 \pm 2.928$ & $79.50 \pm 3.304$ & 0.8387 \\
Fasting blood glucose (mmol/L), mean (SD) & $5.249 \pm 0.1281$ & $5.310 \pm 0.1240$ & 0.7694 \\
Fasting insulin (pmol/ml),mean(SD) & $9.664 \pm 1.731$ & $6.883 \pm 1.176$ & 0.3151 \\
Systolic blood pressure (mmHg),mean(SD) & $122.8 \pm 10.18$ & $107.5 \pm 3.227$ & 0.3304 \\
Diastolic blood pressure (mmHg),mean(SD) & $73.75 \pm 3.063$ & $66.75 \pm 3.119$ & 0.1842 \\
Total cholesterol (mmol/L),mean(SD) & $4.983 \pm 0.3528$ & $4.820 \pm 0.5057$ & 0.7965 \\
Triglyceride (mmol/L),mean(SD) & $1.281 \pm 0.09644$ & $0.9400 \pm 0.1802$ & 0.0945 \\
Low density lipoprotein (mmol/L),mean(SD) & $2.414 \pm 0.1042$ & $2.425 \pm 0.2925$ & 0.9646 \\
High density lipoprotein (mmol/L),mean(SD) & $1.449 \pm 0.09990$ & $1.593 \pm 0.1441$ & 0.4282 \\
\hline
\end{tabular}


Fig. 1. Comparison of Global DNA methylation in phlegm-dampness constitution (PC) with balanced constitution (BC) groups. (A) Filter workflow of Illumina 450k Infinium Methylation BeadChip. (B) The overall correlation plot between the beta values of all CpG sites in PC and BC. DNA methylation was measured as the beta value ranging from 0 (unmethylated) to 1 (completely methylated). All available probes (316356 CpG sites) are shown. (C) Volcano plot of all CpG methylation $\beta$-values. The data for all available probes (316356 CpG sites) are plotted as $\beta$-values versus the $-\log 10$ of the P-values. Thresholds are shown as dashed lines. Genes selected as significantly different are highlighted as red dots.

16). The fractions of low methylated $\mathrm{CpG}$ sites $(<25 \%)$ in the $\mathrm{PC}$ and $\mathrm{BC}$ groups were $30.27 \%$ and $30.32 \%$, respectively. The PC group showed $49.16 \%$ highly methylated CpG sites fraction ( $>75 \%$ ) compared with $47.89 \%$ in $\mathrm{BC}$ group. As shown in Fig. 2A, the average global levels of DNA methylation were similar between PC and BC subjects.

Using Student's t-test to compare each of the CpG sites individually, 2252 differentially methylated probes were detected in the 316, 356 included probes, while 1658 differential probes were located by the Wilcoxon signed rank test. The overlapping 1011 probes were identified to be significantly $(\mathrm{p}<0.01)$ differentially methylated between PC and BC individuals (Fig. 1C, Fig. 2B). The $\Delta \beta$ was also considered as a criterion of DMP identification, 288 of these 1011 probes met the threshold of $|\Delta \beta|$ $\geq 0.05$, including 187 hypermethylated and 101 hypomethylated ones (Fig. 2B), and the top 25 DMPs, sorted by the $\Delta \beta$, were listed in Table 2.

According to the analysis of these 288 DMPs, the subjects in the PC group exhibited higher methylation levels than those in the BC group (Fig. 2C). The proportions of 288 DMPs measured by location relative to $\mathrm{CpG}$ island regions and gene regions are presented in Fig. 3A and 3B, respectively.

Most of the hypermethylated DMPs are located in the Open Sea, followed by the North Shore and the South Shore. For the hypomethylated DMPs, CpG islands were ranked first and were followed by the North Shore and the Open Sea. In terms of gene regions, both hyper and hypomethylated DMPs were predominantly located at the promoters, included TSS1500, TSS200, UTR5 and 1stExon. Supervised hierarchical clustering based on the data of 288 DMPs demonstrated that there were distinctly different DNA methylation patterns between subjects in the PC and BC groups, which are displayed as a heatmap in Fig. 3C. 
Table 2. Top 25 differently methylated $\mathrm{CpG}$ sites based on the $\Delta \beta(\mathrm{P}<0.01)$

\begin{tabular}{|c|c|c|c|c|}
\hline Illumid & Gene symbol & Gene feature & $\Delta \beta$ & Associated diseases \\
\hline cg21498547 & DLGAP2 & 3'UTR & 0.495 & epithelial cancer, adenocarcinoma, Alzheimer's disease, carcinoma, endometrioid cancer, non-insulin-dependent diabetes mellitus \\
\hline c13925773 & DUSP19 & TSS200 & -0.179 & \\
\hline cg26542412 & TGFBR3 3 & 5'UTR & 0.166 & acute myeloid leukemia, ovarian neoplasia, Duchenne muscular dystrophy,etc. \\
\hline cg26029734 & PAX6 & Body & -0.161 & aniridia, congenital aniridia, irido-corneo-trabecular dysgenesis, aniridia, etc. \\
\hline cg16155081 & TAF1B & TSS1500 & -0.149 & epithelial cancer, adenocarcinoma, carcinoma \\
\hline cg04066190 & TFAP2A & Body & -0.149 & branchiooculofacial syndrome, epithelial cancer, etc. \\
\hline cg00818680 & TMCO7 & Body & 0.148 & melanoma, melanoma cancer, non-insulin-dependent diabetes mellitus \\
\hline cg15082992 & -- & -- & -0.142 & -- \\
\hline cg08627825 & --- & -- & -0.141 & incerion \\
\hline cg12891252 & HNRNPF & 5'UTR & 0.141 & infection by HIV-1, endometrioid cancer, endometrioid carcinoma, epithelial cancer, melanoma, melanoma cancer \\
\hline cg17796323 & $\mathrm{N} 4 \mathrm{BP} 3$ & Body & -0.134 & epithelial cancer, adenocarcinoma, breast cancer, carcinoma \\
\hline $\begin{array}{l}\operatorname{cg} 04206742 \\
\operatorname{cg} 14553705\end{array}$ & TGDS & TSS1500 & -0.132 & Catel-Manzke syndrome, productive infection by HIV-1 \\
\hline $\begin{array}{l}\text { cg14553705 } \\
\text { cg15443732 }\end{array}$ & GALR1 & $\overline{\mathrm{TSS} 1500}$ & 0.13 & melanoma cancer, melanoma, neoplasia \\
\hline cg24768902 & PLXDC1 & TSS1500 & 0.129 & cancer, osteosarcoma, melanoma cancer, angiomatosis, hemangioma, squamous cell carcinoma \\
\hline cg23936410 & & & -0.129 & \\
\hline cg12486814 & C1orf192 & TSS200 & 0.126 & melanoma cancer \\
\hline cg21571166 & ZIC1 & 3'UTR & -0.126 & \\
\hline cg10547050 & PHF12 & Body & -0.125 & $\begin{array}{l}\text { endometrioid cancer, endometrioid carcinoma, epithelial cancer, infection by HIV-1 } \\
\text { type IIC Usher syndrome melanoma cancer melanoma epithelial canceretc. }\end{array}$ \\
\hline cg05033239 & GPR98 & $\begin{array}{l}\text { Body } \\
\text { TSS150.TSP20 }\end{array}$ & 0.125 & $\begin{array}{l}\text { type IIC Usher syndrome, melanoma cancer, melanoma, epithelial cancer,etc. } \\
\text { megalencephalic leukoencephalopathy with subcortical cysts } 1 \text { adenocarcinoma }\end{array}$ \\
\hline $\operatorname{cg} 07310406$ & MLC1 & TSS1500;TSS200 & -0.124 & $\begin{array}{l}\text { megalencephalic leukoencephalopathy with subcortical cysts } 1 \text {, adenocarcinoma, etc. } \\
\text { adenocarcinoma epithelial cancer rheumatoid arthritis }\end{array}$ \\
\hline $\begin{array}{l}\text { cg22151644 } \\
\text { cg25373372 }\end{array}$ & $\begin{array}{c}\text { HOXC4 } \\
\text { FAM46A }\end{array}$ & 5'UTR & $\begin{array}{r}0.123 \\
-0.123\end{array}$ & $\begin{array}{l}\text { adenocarcinoma, epithelial cancer, rheumatoid arthritis } \\
\text { adenocarcinoma, epithelial cancer, neoplasia, tumor }\end{array}$ \\
\hline cg26345888 & DAB1 & TSS200 & -0.121 & a cancer, melanoma, adenocarcinoma, epithelial cancer, schizophrenia, amyotrophic lateral sclerosis, non-insulin-dependent diabetes mellitus \\
\hline
\end{tabular}

Fig. 3. Analysis of 288 DMPs between PC and BC groups. (A) Proportions of 288 DMPs measured by location relative to $\mathrm{CpG}$ isle regions. The six categories of CpG island, North Shelf, North Shore, Open Sea, South Shelf and South Shore are identified according to Illumina $450 \mathrm{~K}$ annotation. (B) Proportions of 288 DMPs measured by functional location relative to gene regions. The regions of $200 \mathrm{bp}$ and 200 to $1500 \mathrm{bp}$ upstream of transcription starting site are categorized as TSS 200 and TSS 1500, respectively. The other five groups are 5' UTR, first exon,

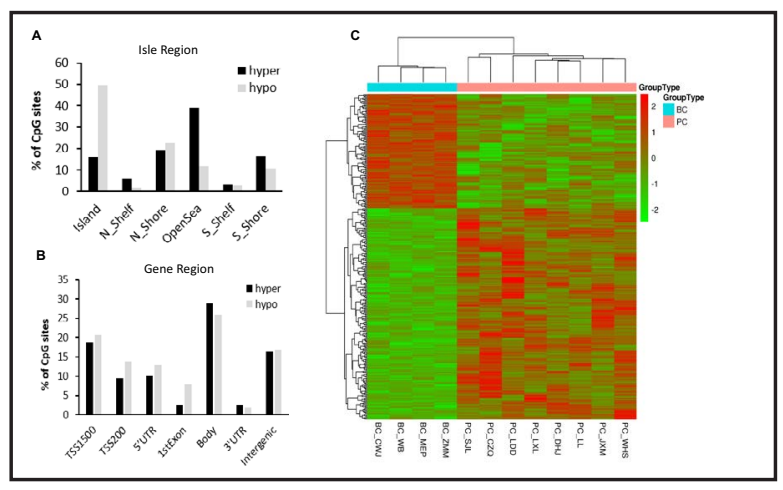
gene body, 3' UTR, and intergenic regions. (C)

Heatmap of the 288 DMPs between PC and BC groups. Columns represent the samples (8 PC subjects and $4 \mathrm{BC}$ subjects) and each row represents a $\mathrm{CpG}$ site. Higher methylation levels are shaded in red and lower levels are in green. The dendrogram shows the results of unsupervised hierarchical clustering of the 288 CpG sites, which separates PC subjects from BC subjects distinctly.

Fig. 4. Manhattan plot and Quantilequantile (QQ) plot. (A) Manhattan plot showing global distribution of DMPs according to the chromosomes sequence. The $\mathrm{x}$-axis represents chromosomes; the y-axis represents - $\log 10$ (p-values). DMPs above the blue line represent a genome-wide significance $(\mathrm{p}<1 \mathrm{e}-04)$. (B) QQ plot of p-values from all 316356 probes. The $\mathrm{x}$-axis shows the expected $\log 10$ (p-value). The $\mathrm{y}$-axis displays

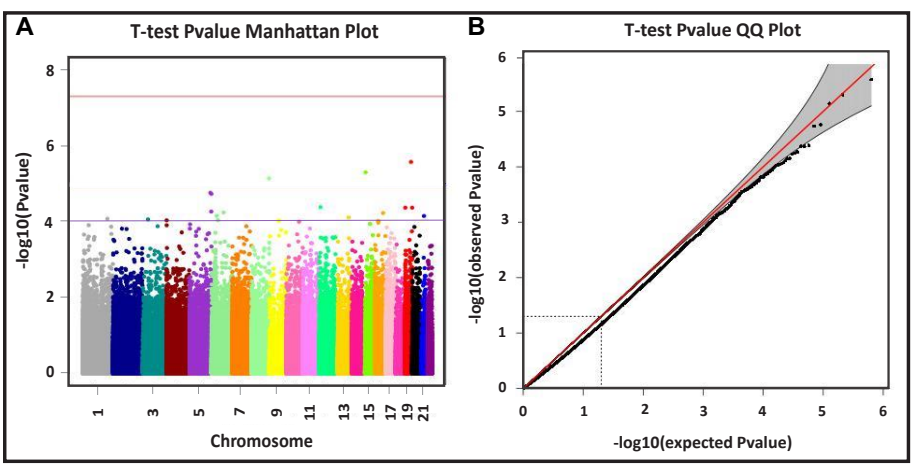
the observed $-\log 10$ (p-value). The red line indicates the expected distributions under the hypothesis. The gray shaded region represents the $95 \%$ confidence interval.

\section{DMPs between $P C$ and $B C$ groups significantly associated with metabolic diseases}

A global view of all included CpG sites is presented in a Manhattan plot (Fig. 4A), indicating that the significant DMPs were mainly located on chromosomes 5, 6, and 19. A list of 256 genes, including 169 hypermethylated and 87 hypomethylated genes, were mapped based on the 288 DMPs. IPA analysis was used to investigate the associated top diseases and bio- 
logical functions of these differentially methylated genes. As shown in Table 3, hypermethylated genes were mainly related to cancer, gastrointestinal disease, cell morphology and lipid metabolism. Hypomethylated genes were mainly related to dermatological diseases, immunological and inflammatory diseases (Table 4). Further gene pathway analysis indicated that the hypomethylated genes were enriched in the pathways related to immune responses (Cytotoxic T lymphocyte-mediated apoptosis of target cells) and metabolism (D-glucuronate degradation I, Glutamate degradation III). The hypermethylated genes were enriched in pathways, including acyl carrier protein metabolism, super pathway of cholesterol biosynthesis II, tetrahydrobiopterin biosynthesis, VDR/RXR activation, and lysine degradation, that were all involved in various metabolic processes (Fig. 5A).

Some genes, which were mapped by top DMPs with the most distinct $\Delta \beta$, were related to the metabolism according to the gene function annotation. In Table 5, we showed that DLGAP2 and DAB1 were involved with diabetes mellitus [25, 26]; HOXC4, HOXC5, and HOXC6 were related to obesity and fat accumulation in differentiated brown adipocytes [27-30]; MECOM was associated with hypertension [31]. It is interesting to note that, of these 288 DMPs, two probes (cg22151644 and cg18473521) were both annotated to gene HOXC4. This implied that this gene might be linked to PC identification.

By the analysis of Manhattan plot in Fig. 4, some specific sites $(\mathrm{p}<1 \mathrm{e}-04)$ were also screened out. The genes mapped by these sites were reported to be closely associated with multiple metabolic diseases (Table 5). For instance, SQSTM1 and FBX09 were related with diabetes mellitus $[32,33]$. GRWD1 and ATP10A were involved with insulin resistance [3436]. SMPD3, ATP10A and CTBP1 were associated with obesity [37-39]. MYO16 was involved with metabolic syndrome [40]. Network visualization and Gene Oncology (GO) analysis of these metabolic-related genes is displayed in Fig. 5B.

\section{Discussion}

This study is the first comprehensive analysis to explore the genome-wide DNA methylation profiles in human PBMCs of PC and BC subjects and to detect the differences between these two constitution types using an Infinium 450K BeadChip assay. The contrasting 
DNA methylation profiles of PC and BC groups provided solid support for the constitution classification system in TCM. Furthermore, this study can verify the reliability of the TCM theory that the healthy individuals within an ethnic population can be divided into distinct groups with corresponding disease risks. Our previous study examined the gene expression patterns of PBMCs isolated from $\mathrm{PC}$ and $\mathrm{BC}$ volunteers, and 355 differentially expressed genes were identified between these two groups [18]. As an ongoing study, this work goes further based on previous studies and confirms the efficacy of TCM constitution classification at the epigenetic level for the first time.

A list of genes obtained from the annotation of 288 DMPs was screened out between PC and BC individuals. IPA results revealed that the molecular and cellular functions of hypermethylated genes, such as B4GALNT1, ST6GALNAC5, DHCR24, and PRKCZ, were significantly associated with lipid metabolism as reported [4143]. The hypomethylated genes (BCL11B, HLA-C, FOXP1, FOXP1) were mainly involved with immunological and inflammatory diseases [44]. It was noteworthy that these molecular features are consistent with previous publications and clinical observations in TCM $[16,44,45]$. In this experiment, the findings were limited by the relatively small numbers of samples. We will try to enlarge the sample scales of PC and BC individuals for further validation. More optimal tissues for obtaining DNA, not only from PBMCs of blood, will also be used for further investigation.

\section{Conclusion}

our present study explored the DNA methylation characteristics of PC for the first time. We identified the DMPs and the corresponding genes associated with obesity, diabetes

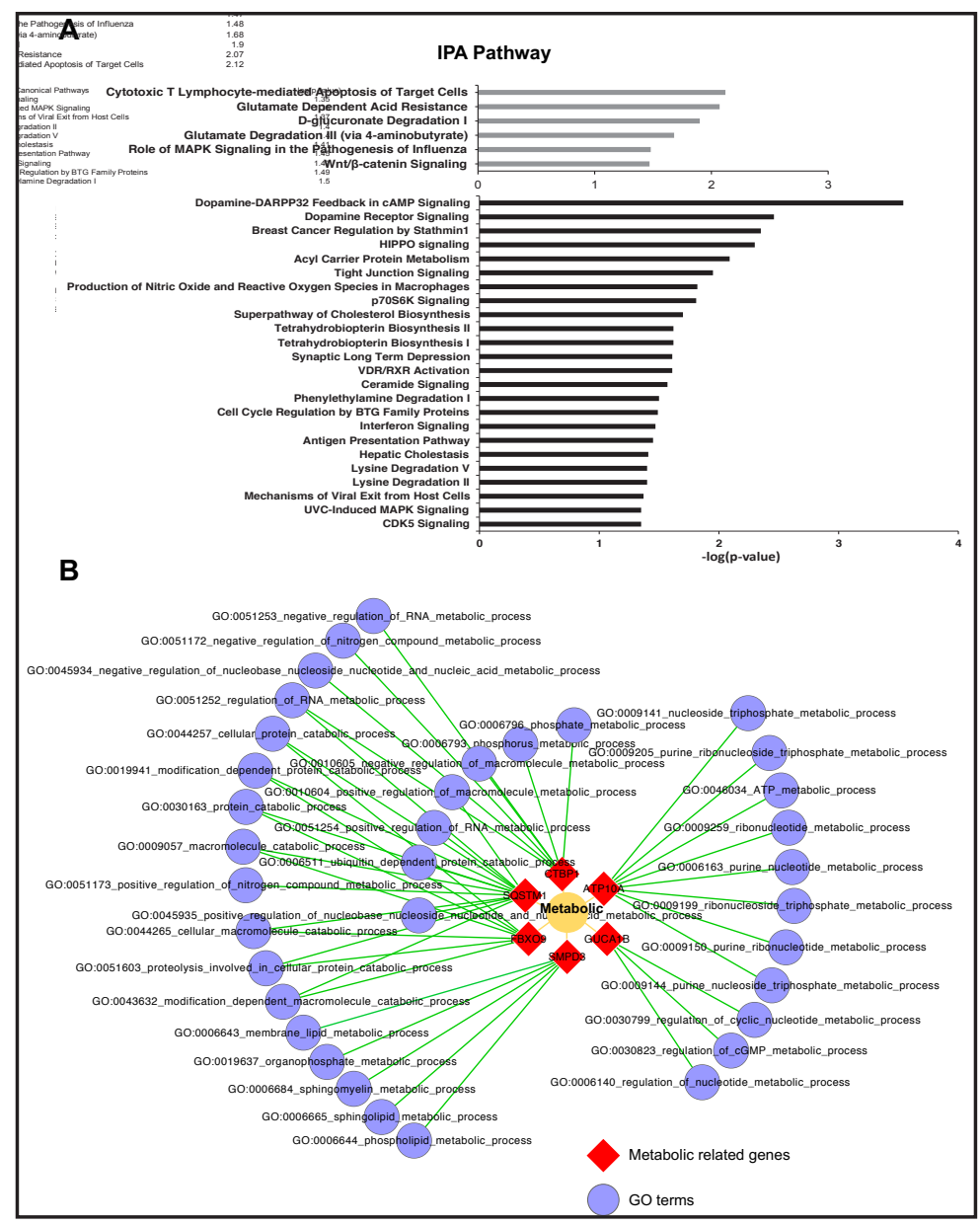

Fig. 5. Pathway analysis and function annotation of genes mapped by DMPs. (A) summary of significant Canonical pathway analysis derived from ingenuity pathway analysis (IPA) for 288 DMPs. The upper gray part and the lower black part indicates the significant pathways for genes with hypomethylation and hypermethylation respectively. The y-axis displays the functional categories that are identified in the analyses. The $\mathrm{x}$-axis demonstrates the significance which is the value of -log (P-value). (B) Network visualization of metabolic related genes and Gene Ontology (GO) terms. The genes were mapped by the top DMPs that were selected by a Manhattan plot analysis. 
mellitus, and other metabolic diseases. These results further support the existing research on molecular biological characteristics of PC. We also provide a possible approach for identifying metabolic disease-susceptible individuals in a healthy population, indicating the objective existence of the subtypes of the healthy population defined by TCM constitution. Apart from the influence of epigenetic mechanisms in the pathogenesis of multiple metabolic disorders [46-49], data from this study suggested that DNA methylation might also play a potential role in the pathological mechanism of PC, which is the incubating stage of multiple metabolic diseases.

\section{Acknowledgements}

This study was supported by the grants from The State Key Program of National Natural Science Foundation of China (No. 81730112), and China Postdoctoral Science Foundation (No. 2017M610830).

\section{Disclosure Statement}

The authors declare no conflicts of interest.

\section{References}

1 Varounis C, Rallidis LS, Franco OH, Lekakis J: Prevalence of metabolic syndrome and association with burden of atherosclerotic disease in patients with stable coronary artery disease. Curr Med Res Opin 2016;32:1175-1181.

2 Yeboah K, Dodam KK, Affrim PK, Adu-Gyamfi L, Bado AR, Owusu Mensah RNA, Adjei AB, Gyan B: Metabolic syndrome and parental history of cardiovascular disease in young adults in urban Ghana. BMC Public Health 2017;18:96.

-3 Guariguata L, Whiting DR, Hambleton I, Beagley J, Linnenkamp U, Shaw JE: Global estimates of diabetes prevalence for 2013 and projections for 2035. Diabetes Res Clin Pract 2014;103:137-149.

4 Vogt H, Hofmann B, Getz L: The new holism: P4 systems medicine and the medicalization of health and life itself. Med Health Care Philos 2016;19:307-323.

$>5$ Nilsson E, Ling C: DNA methylation links genetics, fetal environment, and an unhealthy lifestyle to the development of type 2 diabetes. Clin Epigenetics 2017;9:105.

6 Sayols-Baixeras S, Subirana I, Fernandez-Sanles A, Senti M, Lluis-Ganella C, Marrugat J, Elosua R: DNA methylation and obesity traits: an epigenome-wide association study. The REGICOR study. Epigenetics 2017;12:909-916.

7 Karmaus W: Environmental exposures, epigenetics, and allergy. Toxicol Lett 2015;238:S22-S22.

8 Burris HH, Baccarelli AA, Wright RO, Wright RJ: Epigenetics: linking social and environmental exposures to preterm birth. Pediatr Res 2016;79:136-140.

-9 Romani M, Pistillo MP, Banelli B: Environmental epigenetics: crossroad between public health, lifestyle, and cancer prevention. Biomed Res Int 2015;2015:587983.

10 Wang J, Gong L, Tan Y, Hui R, Wang Y: Hypertensive epigenetics: from DNA methylation to microRNAs. J Hum Hypertens 2015;29:575-582.

11 Leow MK: Environmental origins of hypertension: phylogeny, ontogeny and epigenetics. Hypertens Res 2015;38:299-307.

12 Ronn TS, Volkov P, Gillberg L, Perfilyev A, Jansson PA, Vaag A, Groop L, Nilsson E, Ling C: Age associated DNA methylation changes in adipose tissue is linked to type 2 diabetes. Diabetologia 2015;58:S147-S147.

13 Toperoff G, Kark JD, Aran D, Nassar H, Ahmad WA, Sinnreich R, Azaiza D, Glaser B, Hellman A: Premature aging of leukocyte DNA methylation is associated with type 2 diabetes prevalence. Clin Epigenetics $2015 ; 7: 35$. 


\section{Cellular Physiology Cell Physiol Biochem 2018;45:1999-2008 \begin{tabular}{l|l} 
and Biochemistry & DOI: 10.1159/000487976 \\
\hline Published 2018 The Author(s). Published by S. Karger AG, Basel \\
www.karger.com/cpb
\end{tabular}}

Yao et al.: DNA Methylation Profiles of PC

14 Leslie RD, Dang MA, Hawa MI, Cunningham S, Bradford CM, Lowe R, Paul DM, Libertini E, Lernmark A, Boehm B, Beyan H, Pennington D, Vardhman R, Beck S: DNA methylation variation in type 1 diabetes mellitus. Diabetes 2015;64:A81-A81.

15 Yara S, Lavoie JC, Levy E: Oxidative stress and DNA methylation regulation in the metabolic syndrome. Epigenomics 2015;7:283-300.

16 Wang J, Wang Q Li L, Li Y, Zhang H, Zheng L, Yang L, Zheng Y, Yang Y, Peng G, Zhang Y, Han Y: Phlegmdampness constitution: genomics, susceptibility, adjustment and treatment with traditional Chinese medicine. Am J Chin Med 2013;41:253-262.

17 Zhu Y, Wang Q, Wu C, Pang G, Zhao J, Shen S, Xia Z, Yan X: Logistic regression analysis on relationships between traditional Chinese medicine constitutional types and overweight or obesity. J Chin Integr Med 2010;8:1023-1028.

18 Li L, Feng J, Yao H, Xie L, Chen Y, Yang L, Hou S, Zhao S, Sun R, Wu Y, Bai T, Li Y, Yu R, Wang J, Wang Q: Gene expression signatures for phlegm-dampness constitution of Chinese medicine. Sci China Life Sci 2017;60:105-107.

19 Classification and diagnosis of TCM Constitution. World J Integr Tradit West Med 2009;4:303.

20 Walker DL, Bhagwate AV, Baheti S, Smalley RL, Hilker CA, Sun Z, Cunningham JM: DNA methylation profiling: comparison of genome-wide sequencing methods and the Infinium Human Methylation 450 Bead Chip. Epigenomics 2015;7:1287-1302.

21 Carless MA: Determination of DNA methylation levels using Illumina HumanMethylation450 BeadChips. Chromatin Protoc 2015;143-192.

-22 Moen EL, Zhang X, Mu W, Delaney SM, Wing C, McQuade J, Myers J, Godley LA, Dolan ME, Zhang W: Genomewide variation of cytosine modifications between European and African populations and the implications for complex traits. Genetics 2013;194:987-996.

-23 Maksimovic J, Gordon L, Oshlack A: SWAN: Subset-quantile within array normalization for illumina infinium HumanMethylation450 BeadChips. Genome Biol 2012;13:R44.

-24 Du P, Zhang X, Huang CC, Jafari N, Kibbe WA, Hou L, Lin SM: Comparison of Beta-value and M-value methods for quantifying methylation levels by microarray analysis. BMC Bioinformatics 2010;11:587.

25 Kone M, Pullen TJ, Sun G, Ibberson M, Martinez-Sanchez A, Sayers S, Nguyen-Tu MS, Kantor C, Swisa A, Dor Y, Gorman T, Ferrer J, Thorens B, Reimann F, Gribble F, McGinty JA, Chen L, French PM, Birzele F, Hildebrandt T, Uphues I, Rutter GA: LKB1 and AMPK differentially regulate pancreatic beta-cell identity. FASEB J 2014;28:4972-4985.

-26 Sapkota BR, Hopkins R, Bjonnes A, Ralhan S, Wander GS, Mehra NK, Singh JR, Blackett PR, Saxena R, Sanghera DK: Genome-wide association study of 25(OH) Vitamin D concentrations in Punjabi Sikhs: results of the Asian Indian diabetic heart study. J Steroid Biochem Mol Biol 2016;158:149-156.

-27 Mansego ML, Milagro FI, Zulet MA, Moreno-Aliaga MJ, Martinez JA: differential DNA methylation in relation to age and health risks of obesity. Int J Mol Sci 2015;16:16816-16832.

-28 Cesar AS, Regitano LC, Koltes JE, Fritz-Waters ER, Lanna DP, Gasparin G, Mourao GB, Oliveira PS, Reecy JM, Coutinho LL: Putative regulatory factors associated with intramuscular fat content. PLoS One 2015;10:e0128350.

-29 Keller M, Hopp L, Liu X, Wohland T, Rohde K, Cancello R, Klos M, Bacos K, Kern M, Eichelmann F, Dietrich A, Schon MR, Gartner D, Lohmann T, Dressler M, Stumvoll M, Kovacs P, DiBlasio AM, Ling C, Binder H, Bluher M, Bottcher Y: Genome-wide DNA promoter methylation and transcriptome analysis in human adipose tissue unravels novel candidate genes for obesity. Mol Metab 2017;6:86-100.

-30 Singh S, Rajput YS, Barui AK, Sharma R, Datta TK: Fat accumulation in differentiated brown adipocytes is linked with expression of Hox genes. Gene Expr Patterns 2016;20:99-105.

-31 Sung YJ, de Las Fuentes L, Schwander KL, Simino J, Rao DC: Gene-Smoking interactions identify several novel blood pressure loci in the framingham heart study. Am J Hypertens 2014;28:343-354.

-32 Hua F, Li K, Yu JJ, Hu ZW: The TRIB3-SQSTM1 interaction mediates metabolic stress-promoted tumorigenesis and progression via suppressing autophagic and proteasomal degradation. Autophagy 2015;11:1929-1931.

-33 Lee KW, Kwak SH, Do Koo Y, Cho Y-K, Lee HM, Jung HS, Cho YM, Park YJ, Chung SS, Park KS: F-box only protein 9 is an E3 ubiquitin ligase of PPAR $\gamma$. Exp Mol Med 2016;48:e234.

-34 Santosh Kumar HS, Kumar V, Pattar S, Telkar S: Towards the construction of an interactome for Human WD40 protein family. Bioinformation 2016;12:54-61. 


\section{Cellular Physiology Cell Physiol Biochem 2018;45:1999-2008 \begin{tabular}{ll|l} 
DOI: 10.1159/000487976 & and Biochemistry Published online: March 08, 2018 & $\begin{array}{l}\text { O 2018 The Author(s). Published by S. Karger AG, Basel } \\
\text { www.karger.com/cpb }\end{array}$
\end{tabular}}

Yao et al.: DNA Methylation Profiles of PC

-35 Irvin MR, Wineinger NE, Rice TK, Pajewski NM, Kabagambe EK, Gu CC, Pankow J, North KE, Wilk JB, Freedman BI: Genome-wide detection of allele specific copy number variation associated with insulin resistance in African Americans from the HyperGEN study. PLoS One 2011;6:e24052.

-36 Folmer DE, Elferink RPO, Paulusma CC: P4 ATPases-lipid flippases and their role in disease. BBA-Mol Cell Biol L 2009;1791:628-635.

-37 Kolak M, Westerbacka J, Velagapudi VR, Wågsäter D, Yetukuri L, Makkonen J, Rissanen A, Häkkinen A-M, Lindell M, Bergholm R: Adipose tissue inflammation and increased ceramide content characterize subjects with high liver fat content independent of obesity. Diabetes 2007;56:1960-1968.

-38 Moiola CP, De Luca P, Zalazar F, Cotignola J, Rodríguez-Seguí SA, Gardner K, Meiss R, Vallecorsa P, Pignataro 0, Mazza 0: Prostate tumor growth is impaired by CtBP1 depletion in high-fat diet-fed mice. Clin Cancer Res 2014;20:4086-4095.

39 Sigurdsson MI, Jamshidi N, Jonsson JJ, Palsson BO: Genome-scale network analysis of imprinted human metabolic genes. Epigenetics 2009;4:43-46.

40 Nakatochi M, Ushida Y, Yasuda Y, Yoshida Y, Kawai S, Kato R, Nakashima T, Iwata M, Kuwatsuka Y, Ando M: Identification of an interaction between VWF rs7965413 and platelet count as a novel risk marker for metabolic syndrome: an extensive search of candidate polymorphisms in a case-control study. PloS One 2015;10:e0117591.

41 Irmisch A, Huelsken J: Metastasis: new insights into organ-specific extravasation and metastatic niches. Exp Cell Res 2013;319:1604-1610.

42 Luu W, Zerenturk EJ, Kristiana I, Bucknall MP, Sharpe LJ, Brown AJ: Signaling regulates activity of DHCR24, the final enzyme in cholesterol synthesis. J Lipid Res 2014;55:410-420.

43 Tomasetto C, Alpy F, Drin G, Levine T: The lipid transfer protein STARD3: an architect from inside the cell. FASEB J 2015;29:568.561.

-44 Zheng L-Y, Yang L-L, Li L-R, Jing H-R, Wang J, Wang Q-F, Wang Q: Expressions of TNF-alpha, IL-6, CRP, and MCP-1 in phlegm-damp constitution population detected by multiplexed Luminex assay. Chin J Integr Med 2013;33:920-923.

45 Zhu Y, Wang Q, Dai Z, Origasa H, Di J, Wang Y, Lin L, Fan C: Case-control study on the associations between lifestyle-behavioral risk factors and phlegm-wetness constitution. J Tradit Chin Med 2014;34:286-292.

46 Berg A, Kawasawa YI, Salzberg A, Bixler EO, He F, Liao D: Abstract P260: obesity is associated with DNA methylation in population-based adolescents. Circulation 2015;131:AP260.

47 Moody L, Chen H, Pan YX: Postnatal diet remodels hepatic DNA methylation in metabolic pathways established by a maternal high-fat diet. Epigenomics 2017;9:1387-1402.

48 Yara S, Lavoie J-C, Levy E: Oxidative stress and DNA methylation regulation in the metabolic syndrome. Epigenomics 2015;7:283-300.

49 Han L, Liu P, Wang C, Zhong Q, Fan R, Wang L, Duan S, Zhang L: The interactions between alcohol consumption and DNA methylation of the ADD1 gene promoter modulate essential hypertension susceptibility in a population-based, case-control study. Hypertens Res 2015;38:284-290. 